\title{
Omnidirectional Microscopy by Ultrasonic Sample Control
}

P. Helander ${ }^{1}$, T. Puranen ${ }^{1}$, A. Meriläinen ${ }^{1}$, G. Maconi ${ }^{1}$, A. Penttilä ${ }^{1}$, M. Gritsevich ${ }^{1,2,3}$, I. Kassamakov ${ }^{1}$, A. Salmi $^{1 *}$, K. Muinonen $^{1,2}$, E. Hæggström ${ }^{1}$

${ }^{1}$ Department of Physics, University of Helsinki, P. O. box 64, 00014, Helsinki, Finland

${ }^{2}$ Finnish Geospatial Research Institute FGI, Geodeetinrinne 2, 02430 Masala, Finland

${ }^{3}$ Institute of Physics and Technology, Ural Federal University, 620002 Ekaterinburg, Russia

* Corresponding author, email: ari.salmi@helsinki.fi

\begin{abstract}
Omnidirectional microscopy (OM) is an emerging technology capable of enhancing the threedimensional (3D) microscopy widely applied in life sciences. In OM, precise position and orientation control of the sample are required. However, current OM technology relies on destructive, mechanical methods to hold the samples, such as embedding samples in gel or attaching them to a needle to permit orientation control. A non-contacting alternative is to levitate the sample. Up until now levitation methods have lacked orientation control. We enable omnidirectional access to the sample by introducing a method to control acoustic levitation that provides precise orientation control. Such control around three axes of rotation permits rapid imaging of the sample from any direction with a fixed camera and subsequent 3D shape reconstruction. The control of non-spherical particles is achieved using an asymmetric acoustic field created with a phase-controlled transducer array. Our technology allows robust 3D imaging of delicate samples and their study in a time-lapse manner. We foresee that the described method is not limited to microscopy and optical imaging, but is also compatible with automated sample handling, light-sheet microscopy, wall-less chemistry, and non-contacting tomography.
\end{abstract}

Three-dimensional (3D) microscopy is used increasingly in many scientific fields, e.g. in research of biological structures [1-7], replacing the centuries-old 2D technologies. Existing imaging methods require orientation control of the sample either for tomographic angle scanning or aligning of the sample for optimal illumination and imaging. The orientation control is limited to a single axis and it is invasive since it relies on suspending the sample in gel or on gluing the sample to a holder [1-8]. The capability of accessing the sample from many directions enables omnidirectional microscopy (OM) [1,2], which gives a comprehensive view of the sample. 
Acoustic levitation allows non-contacting and precise manipulation of samples without setting strong limitations on the material properties of the sample: Any millimeter size liquid or solid sample can be levitated, as opposed to the cases of magnetic and optical levitation [9]. Acoustic levitation has been used with light scattering [10,11,12], Raman scattering [13], X-ray scattering [14], mass spectrometry [15], and other techniques [16,17].

The first acoustic levitators, which employed a single standing wave between a transmitter and reflector, were capable of applying forces predominantly along one axis $[9,18]$. In recent years, phased array levitators have made it possible to control and manipulate the acoustic field which has enabled sample transport with dynamic fields and limited orientation locking with spatially asymmetric acoustic traps $[19,20,21,22]$.

The current levitators lack precise orientation control of the sample even around a single rotation angle $[19,20,23]$. Attempts have been made to overcome this limitation, but they are limited to spinning $[20,24,25,26]$ or locking around the $z$-axis $[19,27,28,26,23]$. The current state of the art in orientation control demonstrated locking in a single orientation with the angular range limited to a single plane [19]. Full three axis control of the orientation would be a crucial step for any levitation method to be used in practical applications. We show non-contacting and three-axis sample control and demonstrate 3D surface reconstruction in a setup employing a stationary camera.

To achieve rotational control, we developed an algorithm to precisely align the acoustic trap and we built a custom-made phased-array transducer (PAT) based levitator. The device (Fig. 1d) consists of 444 ultrasonic transducers distributed evenly on the surface of a $\varnothing=15 \mathrm{~cm}$ sphere, split into two hemispheres. The $\varnothing=10 \mathrm{~mm}$ transducers featured a central frequency of $40 \mathrm{kHz}$, and a bandwidth of $1 \mathrm{kHz}$. Custom-built electronics were used to generate sinusoidal signals with amplitude and phase control for each of the 24 channels. The samples were imaged during the levitation to quantify both the levitator performance and the samples.

In acoustic levitation studies, the transducers of the levitator array are often assumed to be identical. In our approach the transducers, however, were grouped into channels with different shape and size to allow simpler driving electronics with less channels. We reasoned that calibrating the levitator, i.e. measuring the acoustic pressure against the driving voltage, is easier than determining the exact positions of the transducers. This approach also eliminates errors originating from differences between transducers. We calibrated the levitator array by measuring the acoustic pressure at the intended levitation spot. The pressure-voltage curve and phasevoltage curve were measured for each channel. These curves were used to obtain the compensated drive voltage amplitude and phase for each channel. In addition to the amplitude and phase, each wave front direction at the levitation spot was measured by moving the microphone and by studying the phase shift. The measured phase shift was linearly fitted, and 
the corresponding wave vectors were calculated for each channel. The approach takes into account possible reflecting waves as it maps the actual pressure field instead of merely using the calculated field based on a point source approximation for the transducers. Further details are described in the Supplementary Material.

Phased-array transducers (PATs) can be used to create sophisticated acoustic field configurations. However, in addition to the increased engineering effort, PATs require a method for determining the drive parameters, i.e., channel-wise amplitude, phase, and frequency. This algorithm is important in acoustic levitation, especially when pursuing precise orientation control. It is insufficient to merely focus the sound onto the levitation spot: the waves must interfere correctly to create the pressure gradient pointing in the desired direction.

The preceding method [20] for calculating the drive parameters first formulates the Gor'kov potential [29] as a function of the phases, amplitudes, and a single frequency in the intended levitation spot. It then minimizes the potential and applies an additional condition to achieve the preferred orientation. This was done using an iterative optimizer with the objective function including the value of the Gor'kov potential and its weighted spatial second derivatives. The drawbacks of this approach include the need for choosing the weights and that it foremost considers forces, not torques.

Our method removes these limitations and enables precise orientation control. The solution relies on the fact that it is possible to approximate the problem in a linear form and to faithfully apply the alignment conditions to the pressure field (constraint) as opposed to the minimizer method that compromises between the conditions (objective). The Gor'kov potential for a hard sphere, with volume $V_{p}$, is

$$
U=V_{p}\left(\frac{1}{2 \rho c^{2}}\left\langle P^{2}\right\rangle-\frac{3}{4} \rho\left\langle V^{2}\right\rangle\right),
$$

where $P$ is acoustic pressure, $V$ is acoustic particle velocity, \langle\rangle means time averaging, $\rho$ and $c$ are the density of air and the speed of sound in air, respectively. For three-axis rotation control, the potential must be asymmetric in three dimensions. Then, if the levitated sample is asymmetric, e.g. a three-axis ellipsoid, there will be a definite energy minimum for a single fixed orientation. One way to achieve an asymmetric acoustic field is to have two perpendicular (directions $\boldsymbol{b}_{z}$ and $\boldsymbol{b}_{y}$, Fig. 1a) standing waves with 90-degree phase difference and an amplitude ratio $\alpha \neq 1$. Such a field is presented in Fig. 5a for $\alpha=0.4$. A similar approach is used in [27] for rotation around one axis using two transducers and reflectors. Here we generalize this method to three dimensions and an arbitrary number of transducers. Other ways for creating an asymmetric field include using two separate fields mixed in time [19] or using two separate frequencies [30]. We chose to use the phase as the other two methods introduce a beat frequency effect which could reduce the acoustic trap stability. 
In three dimensions all the necessary conditions for creating such a field can be written as two equations $p=0$ and $\boldsymbol{\nabla} p \propto \boldsymbol{b}_{z}+i \alpha \boldsymbol{b}_{y}$ where $p$ is the complex-valued pressure field evaluated in the levitation spot. Having $p=0$ ensures that there is a potential minimum that fixes the sample location whereas $\nabla p$ defines the alignment of the field and thus the sample orientation. These criteria can be written as eight scalar equations. The conditions define an asymmetric vortex field whose Gor'kov potential is similarly asymmetric

$$
p_{\text {vortex }}=p_{0} e^{-i \omega t}\left(\sin \left(\mathbf{b}_{\mathbf{z}} \cdot \mathbf{x}\right)+i \alpha \sin \left(\mathbf{b}_{\mathbf{y}} \cdot \mathbf{x}\right)\right) \text {, }
$$

where $\mathbf{x}$ is the displacement from the levitation spot and $\omega=2 \pi f$ is the angular frequency. The total pressure $p$ can be written as the superposition of the array element pressures. Because the region of interest is small, we can approximate each pressure wave as a plane wave with a complex amplitude $p_{n}$ and wavevector $\mathbf{k}_{\mathbf{n}}$

$$
p(\mathbf{x}, t)=e^{-i \omega t} \sum_{n} p_{n} e^{i \mathbf{k}_{\mathbf{n}} \cdot \mathbf{x}}
$$

Now the original conditions yield $\sum p_{n}=0$ and $\sum p_{n} \mathbf{k}_{\mathbf{n}} \propto \mathbf{b}_{\mathbf{z}}+i \alpha \mathbf{b}_{\mathbf{y}}$, which are linear equations and therefore straightforward to tackle. The number of degrees of freedom of a PAT driving state is high: each channel has two degrees of freedom (amplitude and phase) and the levitator PATs can have hundreds of channels. Hence, the solution to the proposed equations is not unique. Singular value decomposition (SVD) provides a fast and robust method to find a feasible solution. This solution minimizes the intensity of the array elements $\sum\left|p_{n}\right|^{2}$ which is beneficial since the solution employs all transducers evenly, i.e., all transducers are loaded within their rated power limits as the opposite would render the solution unusable. This allows emitting sufficient acoustic power and hence permits levitation of relatively heavy samples. A similar optimization scheme has routinely been used in sound field synthesis [31]. By using the approach to fix also the pressure gradient, we can expand its applicability to controlled acoustic levitation. Details for constructing the necessary matrix equation are found in the Supplementary Material.

The proposed method is scalable since inverting a 1000 row matrix is trivial. The method appears to be robust since it works reliably even with as few as 24 channels. An advantage is that the SVD computation is required only once for each levitation spot after which each orientation can be computed instantly with simple matrix multiplication. In addition to being scalable with regards to the number of transducers, the proposed method can be extended to multiple levitation spots, multiple frequencies, and simultaneously pulsed signals. These latter modes of operation can be used to strengthen the trapping along the third direction.

The sample orientation was determined in post-analysis with a customized structure-frommotion (SfM) pipeline based on Mapillary OpenSfM. A standard SfM algorithm [32] detects common features in a set of images and stitches them together into a 3D presentation, 
simultaneously finding the camera position for each image. In our study, the sample rotation with a fixed camera is analogous to a situation where the camera moves around a static sample. We modified the OpenSfM pipeline to use the intended levitation orientation as weak a priori information for the camera position. After reconstruction, the detected position and camera tilt were converted back to the sample orientation.

Each reconstruction was carried out using $3 \times 120$ images taken around the main rotation axis in 3-degree steps. After the reconstruction, the rest of the images from the stability and off-axis studies were added to this initial fit. This was done both to speed up the process and to alleviate the fact that matching multiple nearly identical pictures causes the model to diverge.

The challenges faced by the SfM method includes not having an ideal Lambertian reflection and not having texture on the sample surface. To make the reconstruction more robust a few spots were added to the test sample surface with a marker.

The SfM algorithm provides a 3D point cloud of features that it has matched. The 3D models of the samples were reconstructed from these point clouds using a mesh processing software. The cloud was filtered, and Gaussian surface reconstruction was done to derive the 3D geometry. Further details can be found in Supplementary Material.

The orientation stability was determined around all three main axes, followed by a path featuring off-axis orientations, Figure 2. Each sample was rotated around all axes with 3-degree steps (Supplemental Movie 2). The average absolute angular deviation from the desired orientation was $3^{\circ} \pm 2^{\circ}$, achieving a highest precision for the pitch angle of $2^{\circ} \pm 1^{\circ}$, (Fig. 2a). The angular instability ( $1-\sigma$ level) over 60 seconds was $1^{\circ}$ (Fig. $2 \mathrm{~b}$ ) with a maximum error of $3^{\circ}$. Finally, we demonstrated the ability to rotate the levitated sample around all axes simultaneously (Fig. 2c) by going through a set of subsequent randomly chosen orientations. The target orientations were achieved to within ten degrees (median), the deviation is higher than the one quoted previously because uncertainty now adds up over the three angles concurrently. Videos of each data set are presented as Supplementary Material (Supplemental Movies 2, 3).

We performed full 3D reconstructions of two sample classes: a control sample and a post mortem fly (Musca domestica). The control sample was rotated around all axis, whereas the fly was rotated around the z-axis (Fig. 3, Supplemental Movie 1), the sample sizes were $4 \mathrm{~mm}$ and $3 \mathrm{~mm}$, respectively. The smallest distinguishable features were $50 \mu \mathrm{m}$. This is the first 3D reconstruction from all angles where the sample was held in a non-contacting manner during imaging.

The torque simulations were carried out using COMSOL Multiphysics ${ }^{\circledR}$ 5.2. The sample geometry, obtained with SfM, was used in the simulations. The results show that the acoustic trap is stable against rotation around any axis (Fig. 1c) and the trap stiffness is on order of $1 \mathrm{nNm} / \mathrm{deg}$ (for pitch). The stiffness changes drastically between the different rotation axes, e.g. the yaw is ten 
times stiffer than the roll and pitch. The stiffness ratio between the axes can be changed by altering the field asymmetry $\alpha$ [33]. The harmonic force yields theoretically a resonant frequency of $23 \mathrm{~Hz}$ for the control samples against the largest moment of inertia, which matches the $20 \mathrm{~Hz}$ oscillations observed experimentally. The solved scattered pressure field is presented in Fig. 5b. The sample surface was approximated as a hard boundary and the incidence pressure field was approximated as the superposition of two standing waves. Running the simulations in the frequency domain using a real geometry permitted rapid computation and reliable results. Videos of the incident and scattered field as well as details of the simulation are presented in Supplemental Data and Supplementary Material (Supplemental Movie 5).

The time-lapse capability of the method is demonstrated in Fig. 4 (and Supplemental Movie 4), where the evolution of leavening dough is followed during levitation. The stable levitation of the sample permits studying processes over time [34] while causing minimum disturbance. Combined with the capability of setting the orientation, the time-lapse study provides a way to produce four-dimensional (4D) models, that is, 3D models evolving in time.

We introduced a method, based on acoustic levitation that enables non-contacting omnidirectional imaging of a sample. We showed stable and precise orientation control and demonstrated 3D surface reconstruction. The achieved precise sample control can open a new field of non-contacting omnidirectional microscopy. Our method may be especially valuable when combined with existing techniques such as light-sheet microscopy, tomography, automated sample handling, light-sheet microscopy [3] or wall-less chemistry [35]. The current device is limited to asymmetric samples with a longest dimension of $0.5 \mathrm{~mm}$ to $5 \mathrm{~mm}$, this scales one-to-one with the wavelength used.

For the field of acoustic levitation, we expect the proposed asymmetric trap method to become the standard as it is a robust and fast way to achieve precise orientation control. The method can permit active feed back during levitation to damp unwanted sample oscillations [36]. The method can be used in any phased array application where the control over the emitted field characteristics is beneficial. It can be applied for example to study crystallization processes in chemistry or biological experiments in microgravity-like environment. 
Figure 1: Non-contacting Sample Control for Omnidirectional Microscopy
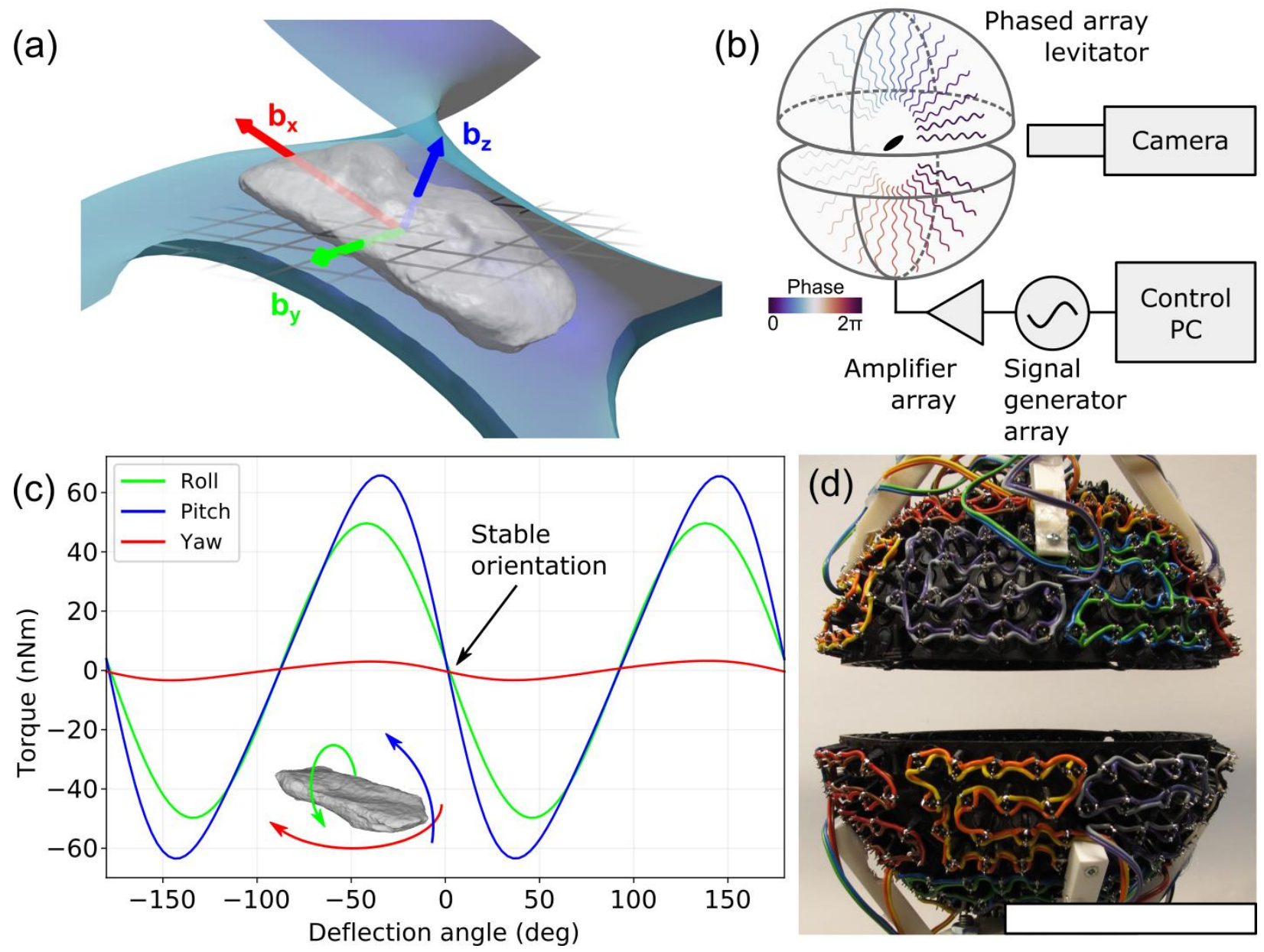

(a), Iso-pressure surface of the acoustic field. The sample, a deformed ellipsoid, aligns with the asymmetric field. (b), Schematic picture of the setup. The ultrasound sources are arranged around the levitation spot. The color represents the phase angle of the wave. (c), Simulated torque shows that the orientation is stable around all three axes. (d), Phased-array levitator consisting of two hemispheres and 444 transducers. Scale bar $10 \mathrm{~cm}$. 
Figure 2: Quantified sample control over three axes of rotation.

(a)

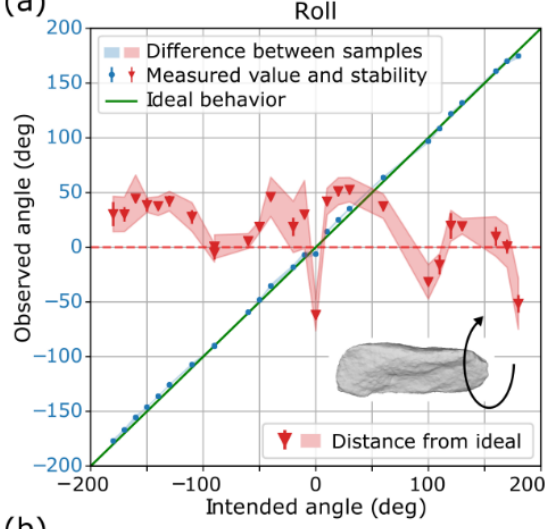

(b)
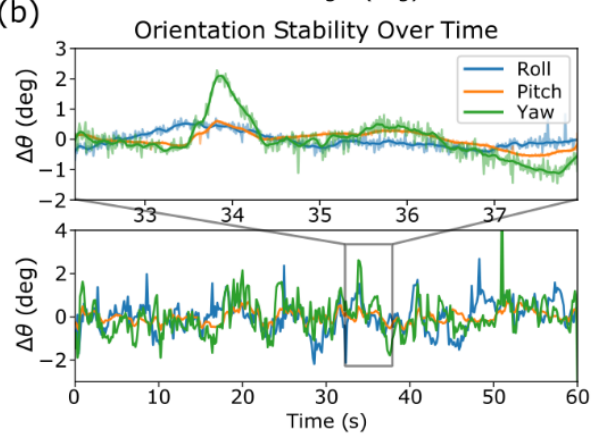

Pitch

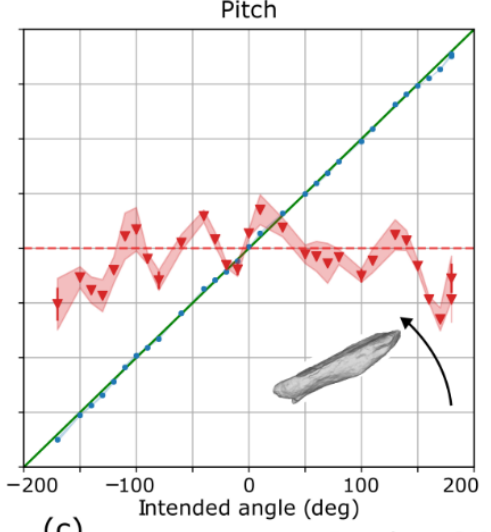

(c)
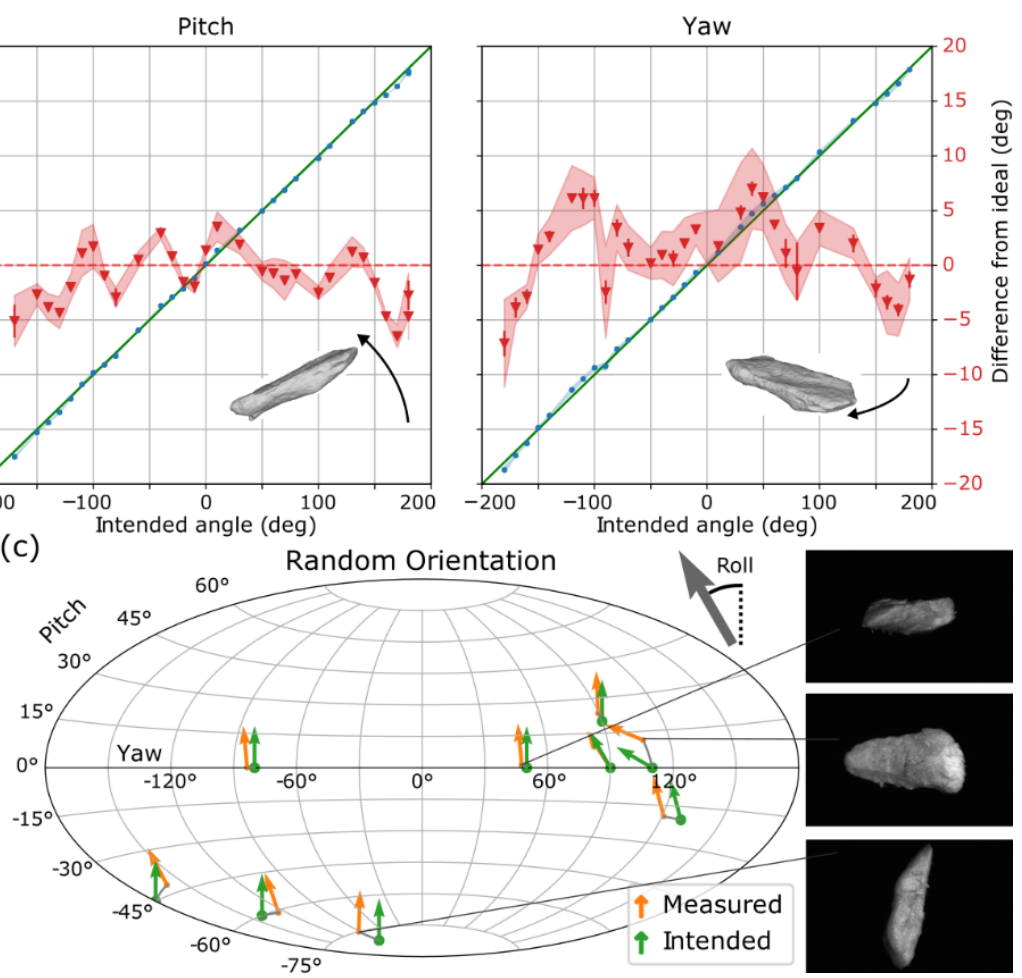

(a), Control of each axis of rotation shown separately. The sample orientation was detected with computer vision. The blue markers show the measurements whereas the red markers show the difference from the ideal (intended) behavior tenfold magnified. The error bar states the variation in time and the red area the variation between samples. (b), Stability over time in one orientation for control sample \#2. (c), Simultaneous rotation about all axes. 
Figure 3: Omnidirectional microscopy

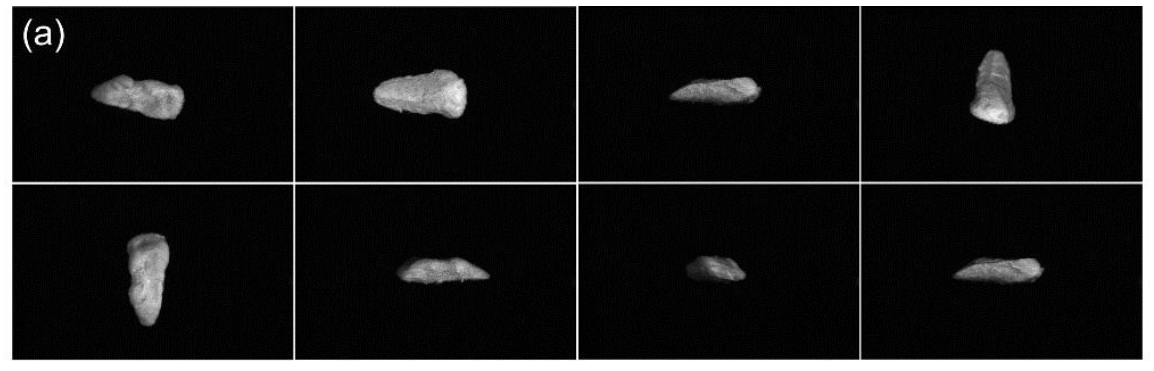

(b)
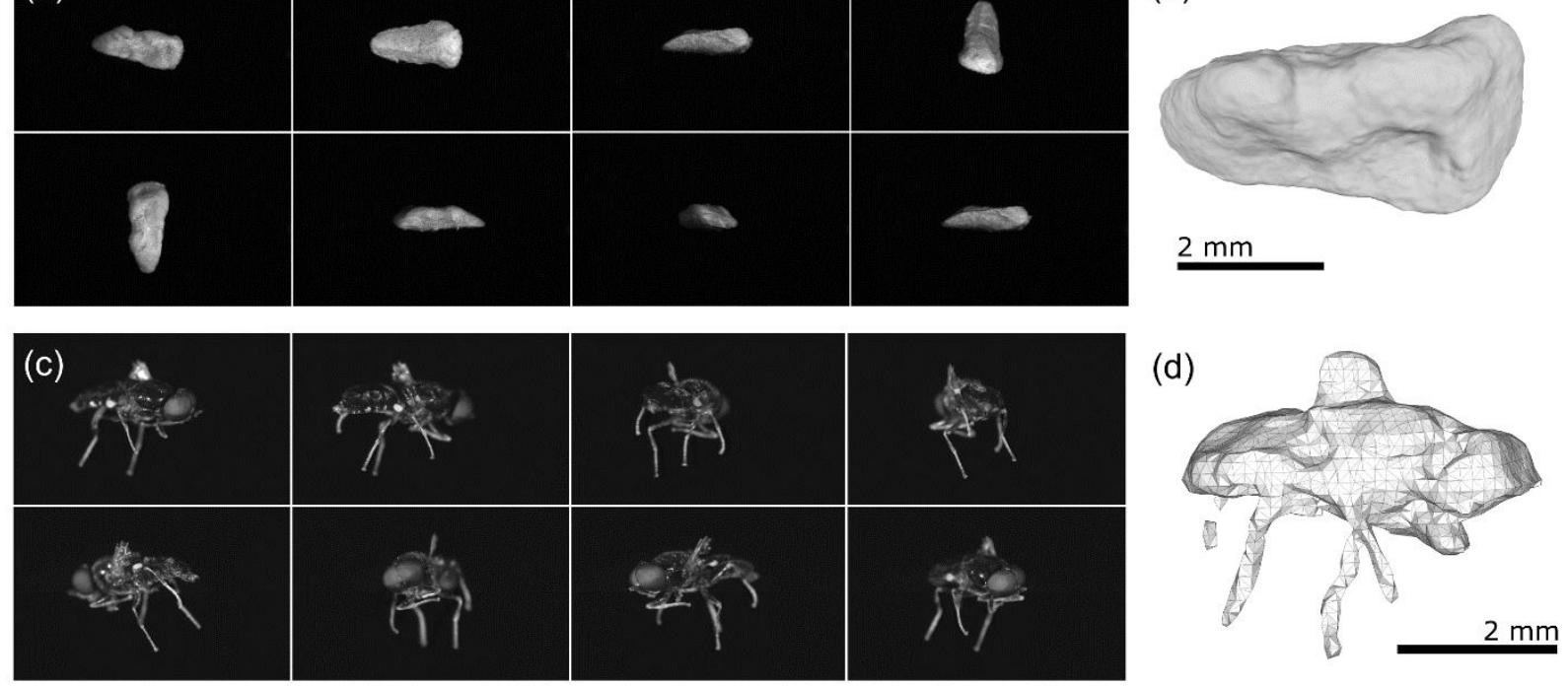

(a), Representative frames from time series of the control sample \#3 that is precisely rotated around three axes. (b), 3D object reconstruction with the structure-from-motion pipeline (300 frames, $5 \mathrm{~min}$ imaging time, $1 \mathrm{~h}$ reconstruction time). (c), Time series of a levitated post mortem fly. (d), Filtered reconstruction of the fly. 
Figure 4: Time-lapse evolution of the levitating sample

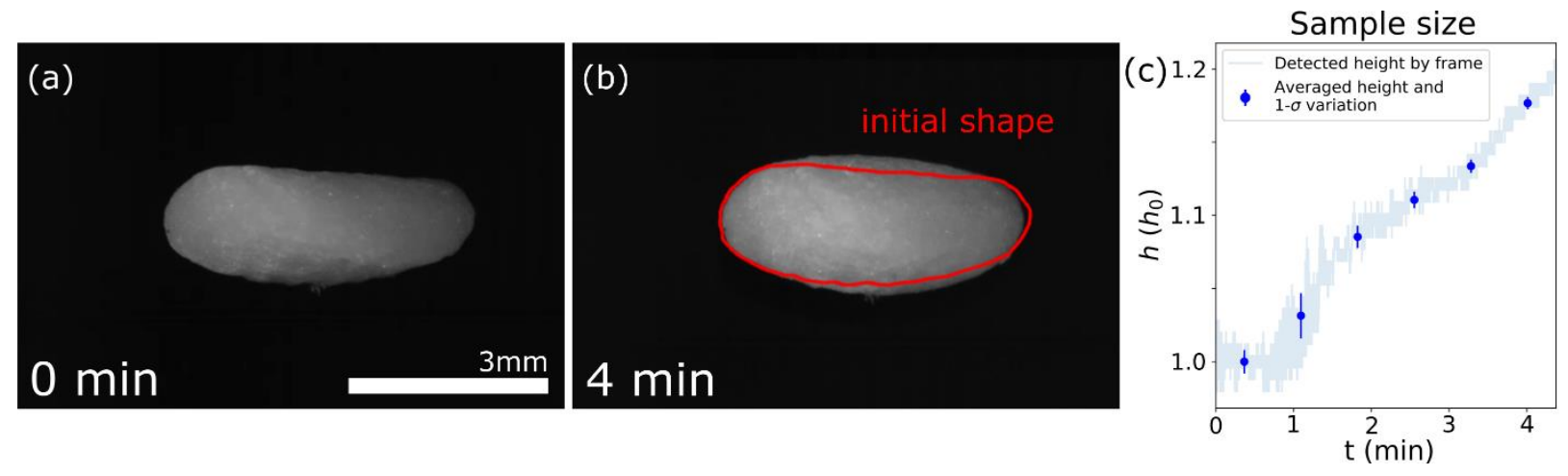

(a), A piece of leavening dough levitated. The shape of the sample changes over time demonstrating ability to obtain a time-lapse. (b), The initial shape overlaid on the inflated sample. (c), The sample height over time. 
Figure 5: Simulated Pressure Field Around the Sample

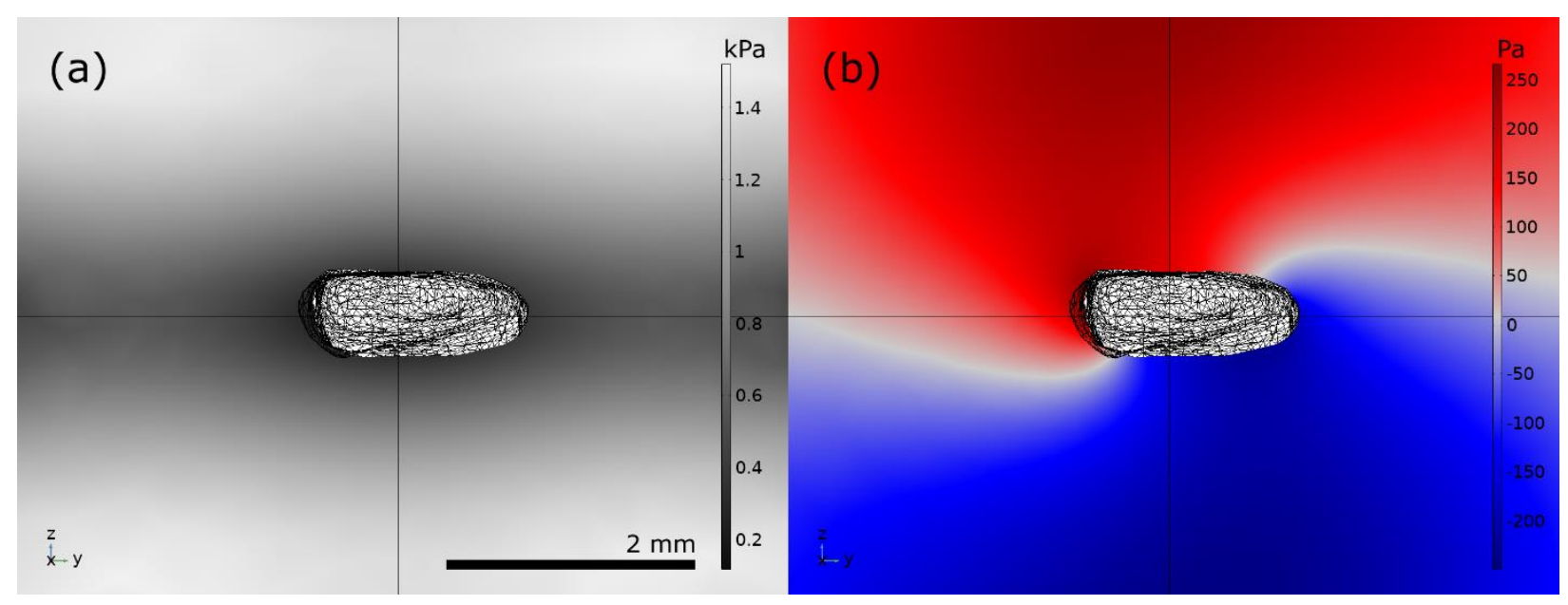

(a), The cross section of incidence pressure field root mean square value and the sample geometry. The equilibrium position of the sample is in the pressure minimum. The smallest pressure gradient and the longest dimension of the sample point out of the image. (b), Instantaneous scattered pressure field. The vortex-like scattered field is created by the vortex-like incidence field. 


\section{Supplementary Material}

Supplementary Material include detailed description of the phase calculation algorithm, the orientation detection pipeline, the experimental setup, and details of the simulations.

\section{Supplementary Video 1}

Caption: Comparison of the levitated fly and its 3D reconstruction. The 3D surface model rotated around the z-axis and the corresponding frame from the levitation video. The frames were aligned with SfM.

Type: Video

Format: MPEG-4

\section{Supplementary Video 2}

Caption: Three-axis control of the levitated control samples. Sped-up videos of each control sample rotated around each axis. Each corner of the video is a separate measurement done successively.

Type: Video

Format: MPEG-4

\section{Supplementary Video 3}

Caption: Off-axis rotations and rotation stability. Control sample \#3 rotated through set of offaxis orientations followed by a clip of control sample \#2 levitated in stable orientation.

Type: Video

Format: MPEG-4

\section{Supplementary Video 4}

Caption: Simulated pressure field. A cross-section (perpendicular to bx) from the numerical pressure field simulations. The incident wave forms a vortex pressure node. Scattered field presented separately.

Type: Video

Format: MPEG-4

\section{Supplementary Video 5}


Caption: Dough time-lapse. Sped-up video of the piece of dough levitated and slowly rotated around z-axis.

Type: Video

Format: MPEG-4

\section{Acknowledgements}

This research was supported by the European Research Council Advanced Grant No. 320773 entitled "Scattering and Absorption of ElectroMagnetic waves in ParticuLate media" (ERC SAEMPL, 2013-2018, PI K. Muinonen). MG acknowledges the Academy of Finland project No. 325806. The authors thank Joni Mäkinen for his help on the use of COMSOL Multiphysics ${ }^{\circledR}$ simulation software.

\section{Contributions}

All authors participated in designing the study and experiments. P. H., T. P., and A. M. built the levitator. P. H. developed the control algorithm and performed the experiments and data analysis. P. H., A. S., T. P., and M. G. wrote the manuscript. All the authors read and commented on the text.

\section{Competing interests}

The authors have filed a patent related to the method for creating the acoustic trap.

\section{References}

1. M. Weber and J. Huisken, “Omnidirectional microscopy”, Nature Methods 9, 656 (2012).

2. B. Schmid, G. Shah, N. Scherf, M. Weber, K. Thierbach, C. P. Campos, I. Roeder, P. Aanstad, and J. Huisken, "High-speed panoramic light-sheet microscopy reveals global endodermal cell dynamics", Nature Communications 4, 1-10 (2013).

3. P. G. Pitrone, J. Schindelin, L. Stuyvenberg, S. Preibisch, M. Weber, K. W. Eliceiri, J. Huisken, and P. Tomancak, “Openspim: an open-access light-sheet microscopy platform”, Nature Methods 10, 598-599 (2013). 
4. M. Fauver, E. J. Seibel, J. R. Rahn, M. G. Meyer, F. W. Patten, T. Neumann, and A. C. Nelson, "Threedimensional imaging of single isolated cell nuclei using optical projection tomography", Optics Express 13, 42104223 (2005).

5. R. Heintzmann and C. Cremer, "Axial tomographic confocal fluorescence microscopy”, Journal of microscopy 206, 7-23 (2002).

6. V. Richter, S. Bruns, T. Bruns, P. Weber, M. Wagner, C. M. Cremer, and H. Schneckenburger, "Axial tomography in live cell laser microscopy”, Journal of biomedical optics 22, 091505 (2017).

7. H. Chen, J. R. Swedlow, M. Grote, J. W. Sedat, and D. A. Agard, "The collection, processing, and display of digital three-dimensional images of biological specimens", in Handbook of biological confocal microscopy (Springer, 1995), pp. 197-210.

8. T. Bruns, S. Schickinger, and H. Schneckenburger, "Sample holder for axial rotation of specimens in 3d microscopy”, Journal of microscopy 260, 30-36 (2015).

9. E. Brandt, “Acoustic physics: suspended by sound", Nature 413, 474 (2001).

10. G. Maconi, A. Penttilä, I. Kassamakov, M. Gritsevich, P. Helander, T. Puranen, A. Salmi, E. Haeggström, and K. Muinonen, "Non-destructive controlled single-particle light scattering measurement", Journal of Quantitative Spectroscopy and Radiative Transfer 204, 159-164 (2018).

11. K. Muinonen, T. Väisänen, J. Martikainen, J. Markkanen, A. Penttilä, M. Gritsevich, J. Peltoniemi, J. Blum, J. Herranen, G. Videen, et al., "Scattering and absorption of light in planetary regoliths", Journal of Visualized Experiments 149 (2019).

12. G. Maconi, P. Helander, M. Gritsevich, A. Salmi, A. Penttilä, I. Kassamakov, E. Haeggström, and K. Muinonen, "4pi scatterometer: a new technique for understanding the general and complete scattering properties of particulate media”, Journal of Quantitative Spectroscopy and Radiative Transfer, 106910 (2020).

13. L. Puskar, R. Tuckermann, T. Frosch, J. Popp, V. Ly, D. McNaughton, and B. R. Wood, "Raman acoustic levitation spectroscopy of red blood cells and plasmodium falciparum trophozoites", Lab on a Chip 7, 1125-1131 (2007).

14. S. Tsujino and T. Tomizaki, "Ultrasonic acoustic levitation for fast frame rate x-ray protein crystallography at room temperature", Scientific reports 6, 25558 (2016).

15. M. S. Westphall, K. Jorabchi, and L. M. Smith, "Mass spectrometry of acoustically levitated droplets", Analytical chemistry 80, 5847-5853 (2008).

16. S. Gutierrez-Ramos, M. Hoyos, and J. Ruiz-Suarez, "Induced clustering of escherichia coli by acoustic fields", Scientific reports 8, 1-8 (2018).

17. M. Sundvik, H. J. Nieminen, A. Salmi, P. Panula, and E. Haeggström, "Effects of acoustic levitation on the development of zebrafish, danio rerio, embryos", Scientific reports 5, 1-11 (2015).

18. R. Whymark, “Acoustic field positioning for containerless processing”, Ultrasonics 13, 251-261 (1975).

19. L. Cox, A. Croxford, B. Drinkwater, and A. Marzo, "Acoustic lock: position and orientation trapping of nonspherical sub-wavelength particles in mid-air using a single-axis acoustic levitator", Applied Physics Letters 113, 054101 (2018). 
20. A. Marzo, S. A. Seah, B. W. Drinkwater, D. R. Sahoo, B. Long, and S. Subramanian, "Holographic acoustic elements for manipulation of levitated objects", Nature Communications 6, 8661 (2015).

21. A. Marzo, M. Caleap, and B. W. Drinkwater, "Acoustic virtual vortices with tunable orbital angular momentum for trapping of mie particles", Physical review letters 120, 044301 (2018).

22. D. Foresti, M. Nabavi, M. Klingauf, A. Ferrari, and D. Poulikakos, "Acoustophoretic contactless transport and handling of matter in air", Proceedings of the National Academy of Sciences 110, 12549-12554 (2013).

23A. Marzo and B. W. Drinkwater, "Holographic acoustic tweezers", Proceedings of the National Academy of Sciences 116, 84-89 (2019).

24. T. Schwarz, P. Hahn, G. Petit-Pierre, and J. Dual, "Rotation of fibers and other non-spherical particles by the acoustic radiation torque", Microfluidics and Nanofluidics 18, 65-79 (2015).

25. S. Kuhn, A. Kosloff, B. A. Stickler, F. Patolsky, K. Hornberger, M. Arndt, and J. Millen, "Full rotational control of levitated silicon nanorods", Optica 4, 356-360 (2017).

26. D. Foresti and D. Poulikakos, "Acoustophoretic contactless elevation, orbital transport and spinning of matter in air”, Physical Review Letters 112, 024301 (2014).

27. T. Schwarz, G. Petit-Pierre, and J. Dual, "Rotation of non-spherical microparticles by amplitude modulation of superimposed orthogonal ultrasonic modes", The Journal of the Acoustical Society of America 133, 1260-1268 (2013).

28. Z. Hong, J. Yin, W. Zhai, N. Yan, W. Wang, J. Zhang, and B. W. Drinkwater, "Dynamics of levitated objects in acoustic vortex fields", Scientific reports 7, 1-7 (2017).

29. L. P. Gor'kov, "On the forces acting on a small particle in an acoustical field in an ideal fluid", in Soviet Physics Doklady 6 (1962), pp. 773-775.

30. T. Puranen, P. Helander, A. Meriläinen, G. Maconi, A. Penttilä, M. Gritsevich, I. Kassamakov, A. Salmi, K. Muinonen, and E. Haeggström, "Multifrequency acoustic levitation", in 2019 IEEE International Ultrasonics Symposium (IUS) (2019), pp. 916-919.

31. O. Kirkeby and P. A. Nelson, "Reproduction of plane wave sound fields", The Journal of the Acoustical Society of America 94, 2992-3000 (1993).

32. J. J. Koenderink and A. J. Van Doorn, “Affine structure from motion”, JOSA A 8, 377-385 (1991).

33. P. Helander, T. Puranen, A. Meriläinen, G. Maconi, A. Penttilä, M. Gritsevich, I. Kassamakov, A. Salmi, K. Muinonen, and E. Haeggström, "Simulating acoustic orientation trapping for stable levitation", in 2019 IEEE International Ultrasonics Symposium (IUS) (2019), pp. 650-653.

34. B. Neumann, T. Walter, J.-K. Heriche, J. Bulkescher, H. Erfle, C. Conrad, P. Rogers, I. Poser, M. Held, U. Liebel, et al., "Phenotypic profiling of the human genome by time-lapse microscopy reveals cell division genes", Nature 464, 721-727 (2010).

35. S. Santesson and S. Nilsson, "Airborne chemistry: acoustic levitation in chemical analysis", Analytical and bioanalytical chemistry 378, 1704-1709 (2004). 
36. A. E. Wallin, H. Ojala, E. Haeggström, and R. Tuma, "Stiffer optical tweezers through real-time feedback control”, Applied Physics Letters 92, 224104 (2008). 


\section{Omnidirectional Microscopy by Ultrasonic Sample Control Supplementary Information}

\section{Algorithm for precise orientation control}

\section{Single spot}

The acoustic radiation force that enables the levitation originates from the momentum carried by the acoustic waves. The effect is described by the Gor'kov potential that is used to calculate forces on small spherical particles. Stable levitation occurs in the pressure nodes of the standing wave where a potential minimum is formed. The Gor'kov potential for a small sphere is expressed using the acoustic pressure $P$, particle velocity $V$, the speeds of sound and densities $c, \rho$ (medium), $c_{0}, \rho_{0}$ (levitated particle), and the volume of the particle $V_{p}$.

$$
\begin{aligned}
U & =V_{p}\left(f_{1} \frac{1}{2 \rho c^{2}}\left\langle P^{2}\right\rangle-f_{2} \frac{3}{4} \rho\left\langle V^{2}\right\rangle\right), \\
f_{1} & =1-\frac{\rho c^{2}}{\rho_{0} c_{0}^{2}} \approx 1 \\
f_{2} & =\frac{1-\frac{\rho}{\rho_{0}}}{1+\frac{\rho}{2 \rho_{0}}} \approx 1 .
\end{aligned}
$$

The net acoustic force that acts on the sphere is the gradient of the potential $\mathbf{F}=-\nabla U$. The acoustic force is proportional to the particle volume $V_{p}$ similar to the gravitational pull therefore the levitability of a sample is determined by its density and not its weight. The equation S1 in air yields:

$$
U \approx V_{p}\left(\frac{1}{2 \rho c^{2}}\left\langle P^{2}\right\rangle-\frac{3}{4} \rho\left\langle V^{2}\right\rangle\right) .
$$

The simplest way to create an ellipsoidal potential is to use two perpendicular standing waves (from directions $\mathbf{b}_{z}$ and $\mathbf{b}_{y}$ ) with different amplitude and 90 degrees phase difference. They create a vortex like pressure node described mathematically as:

$$
\begin{aligned}
& P_{\text {vortex }}(t, \mathbf{x})=P_{0}\left(\sin \left(\mathbf{b}_{z}^{T} \mathbf{x}\right) \cos (\omega t)+\alpha \sin \left(\mathbf{b}_{y}^{T} \mathbf{x}\right) \cos (\omega t-\pi / 2)\right), \\
& p_{\text {vortex }}(t, \mathbf{x})=p_{0}\left(\sin \left(\mathbf{b}_{z}^{T} \mathbf{x}\right)+i \alpha \sin \left(\mathbf{b}_{y}^{T} \mathbf{x}\right)\right) e^{-i \omega t},
\end{aligned}
$$

where $\alpha$ defines the asymmetry of the trap. When $\alpha$ was $[0.3,0.6]$ the trap was most stable. The pressure along $\mathbf{b}_{x}$ is zero thus no trapping force arises along this direction. However, experiments show levitation even in orientations where the weakest trapping is parallel with the gravity $\left(\mathbf{b}_{x} \| \hat{\mathbf{z}}\right)$. This proves there is a force that originates from the finite size of the sample and the velocity term.

Close to the intended levitation spot $\mathbf{x}=0$, the wave fronts can be approximated as traveling plane waves and the total acoustic pressure $p$ is their superposition:

$$
p(\mathbf{x}, t)=e^{-i \omega t} \sum_{n}^{N} p_{n} e^{i \mathbf{k}_{n}^{T} \mathbf{x}},
$$

where the wavevector $\mathbf{k}_{n}$ is along the direction of the wave projected by $n$ :th channel and $p_{n}$ is its complex amplitude. In the experimental setup we have control over $p_{n}$, i.e., the amplitude and phase of each array element. The acoustic field emitted by the array elements must match with the desired vortex field:

$$
\begin{array}{rlrl}
p(t, \mathbf{0}) & =p_{\text {vortex }}(t, \mathbf{0}) & \Rightarrow & e^{-i \omega t} \sum p_{n}=0, \\
\left.\boldsymbol{\nabla} p\right|_{\mathbf{x}=0}=\left.\boldsymbol{\nabla} p_{\text {vortex }}\right|_{\mathbf{x}=0} & \Rightarrow & e^{-i \omega t} \sum p_{n} i \mathbf{k}_{n}=p_{0}\left(\mathbf{b}_{z}+i \alpha \mathbf{b}_{y}\right) e^{-i \omega t},
\end{array}
$$

where Eq. S5 ensures that the point is a pressure node and Eq. S6 aligns the pressure gradients. When the number of transducers exceeds four $(N>4)$, the solution for equations S5 and S6 is not unique. 
Singular value decomposition allows finding a feasible solution that minimizes $\sum\left|p_{n}\right|^{2}$. Applying SVD requires the equations to be written in matrix form:

$$
\begin{aligned}
\left(\begin{array}{ccc}
1 & . . & 1 \\
i \mathbf{k}_{1} & . . & i \mathbf{k}_{N}
\end{array}\right)\left(\begin{array}{c}
p_{1} \\
\cdot \\
\cdot \\
p_{N}
\end{array}\right)=p_{0}\left(\begin{array}{c}
0 \\
\mathbf{b}_{z}+i \alpha \mathbf{b}_{y}
\end{array}\right), \\
M P=b \\
M=U D V^{T} \\
P=V D^{+} U^{T} b .
\end{aligned}
$$

Here $U D V^{T}$ is the singular value decomposition of $\mathrm{M}$, and $D^{+}$is the generalized inverse of the diagonal matrix D. The parameters for achieving the desired orientation are obtained with Eq. S8. However if the total acoustic pressure $p_{0}$ is unrealistically large the solution must be limited to take into account the limitations of the transducers. Since the pressure calibration curves were measured, the maximum available pressure of each channel is known and the solution can be scaled accordingly, as linear downscaling of each channel preserves the field shape.

Equation $\mathrm{S} 8$ shows that calculating $P$ for each new orientation requires only one matrix multiplication $\left(V D^{+} U^{T}\right)(b)$, which is fast. Changing the levitation location requires that one recalculates the SVD.

Weights can be added for each transducer depending on their location and available power. In this study, SVD was used to minimize the squared sum of relative amplitudes $\sum\left|p_{n} / p_{n}^{\max }\right|^{2}$, i.e., the method was weighted with the channel-wise available power. This was done to remove the burden from the channels that did not perform in a standard manner.

\section{The levitator}

\section{Transducers}

The levitator consists of 444 ultrasonic transducers distributed evenly on a surface of $\varnothing=15 \mathrm{~cm}$ sphere, split in two hemispheres. The $\varnothing=10 \mathrm{~mm}$ transducers (MCUST10P40B07RO, Multicomp) featured a central frequency of $40 \mathrm{kHz}$, and a bandwidth of $1 \mathrm{kHz}$. The transducers were grouped in 24 channels (each top and bottom channel actuated 35 transducers, whereas the other channels actuated 17 transducers, Figure S1). Custom-build electronics (based on AD9106, Analog Devices) were used to generate sinusoidal signals with amplitude and phase control for each channel. The signals were amplified (up to $20 \mathrm{Vpp}$ ) with operational amplifiers (LM3886, Texas Instruments). The hemispheres housing the transducers were 3D printed (Ultimaker Original+, material: Polylactic acid).

\section{Multi-channel signal generator}

The 24-channel signal generator was based on the Analog Device AD9106 Digital Direct Synthesis (DDS) chip. This DDS includes four channel outputs with independent phase and amplitude controls. In this design, seven chips were used, six for the drive signals and one for the reference signal. Each DDS was connected and programmed via an SPI-bus, allowing individual control for each chip. The chips allowed preloading the phase/amplitude configuration without disturbing the active output signals and allowed introducing the new configuration simultaneously to all 24 channels.

The DDS main clock signal was first generated by a Si510 crystal (Silicon Labs), oscillating at 100 MHz. This clock was then distributed to each DDS chip by MC100EP11 (ON Semiconductor) differential signal buffer, Fig S2a. Seven buffer chips were chained to create a buffered clock signal to each DDS.

\section{Amplifier stages}

AD9106 chip has a current source DAC output and a trans-impedance conversion is done by a $1 \mathrm{k} \Omega$ resistor and $510 \Omega$ termination resistors. The AC signal is passed to the preamplifier, OPA830, whose output is AC coupled to the main amplifier LM3886 (Fig. S2b). This high-power operation amplifier was chosen for its wide operation voltage and low output impedance. This operation amplifier has a 


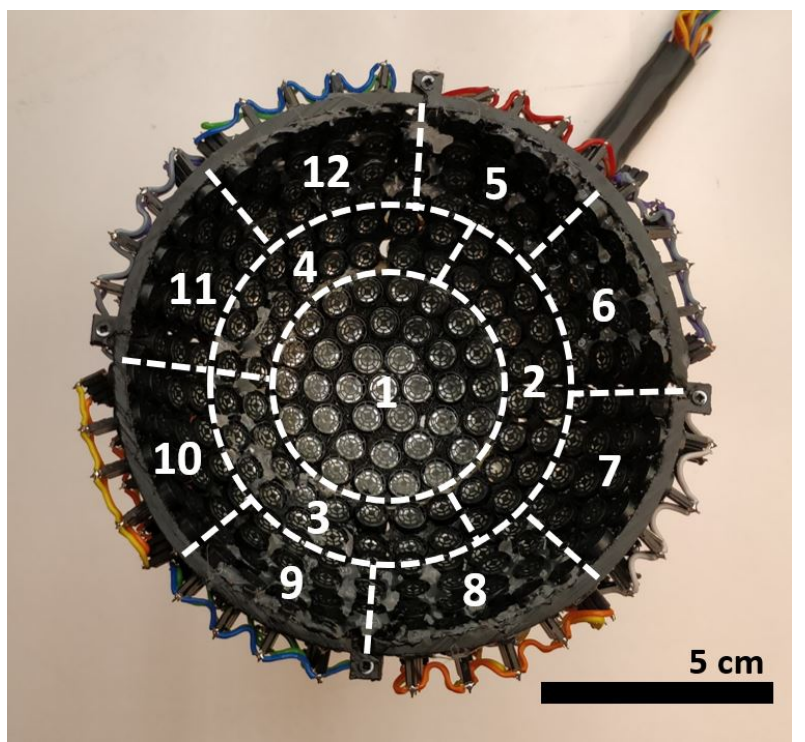

Figure S1: Top view of the other levitator hemisphere. The trasducer groups are labelled.

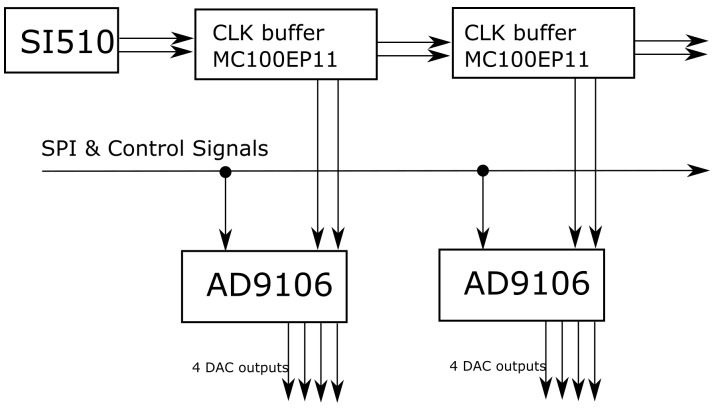

(a) Generator

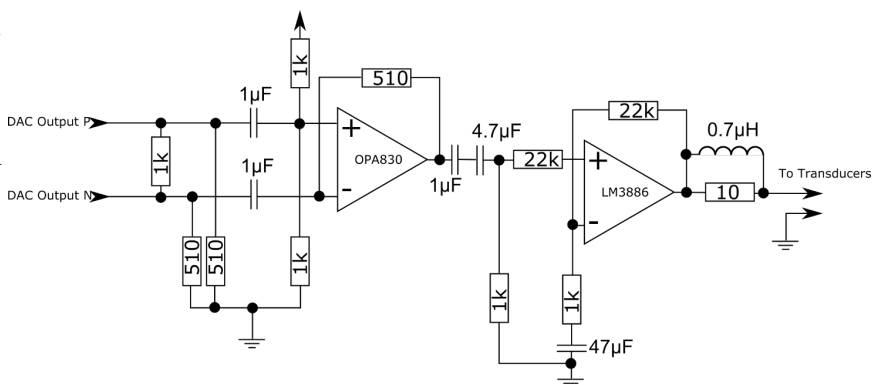

(b) Amplification

Figure S2: Driving electronics

gain-bandwidth-product of $8 \mathrm{MHz}$. In this system, we used 20x gain allowing 400-kHz bandwidth. The LM3886 circuit follows the datasheets non-inverting amplifier circuit design.

\section{Pressure sensing}

The pressure in the levitation spot was measured during the calibration phase with a custom-made microphone: a syringe needle provided a wave guide for a transducer. The small size of the needle tip ensured the sensor to be omni-directional. Although narrow-band, the sensor was suitable for calibrating singular frequencies. The sensing transducer voltage signal was amplified $(37 \mathrm{~dB})$ and bandpassed $(-3$ dB: $30 \mathrm{kHz}-50 \mathrm{kHz}$ ) with a custom-made op-amp (TL074) circuit whose output signal was sampled with Picoscope PS2204A. To ensure measuring a steady state signal the transmitter-receiver system was allowed to ring-in for 100 periods before triggering the measurement. The signal was sampled for 12 periods with 170 samples per period and a sine wave was fitted to it and to the reference signal to obtain the amplitude and phase of the pressure wave. For the calibration the phase shift was measured along each axis with 0.5 -mm step using an xyz-stage (with $10-\mu \mathrm{m}$ resolution).

The amplitude between the different channels varied as much as $50 \%$, this is due to the non-identical transducers and varying number of transducers per channel. Calibration curves are presented in figure S3. It is evident that also phase shift compensation is important. The wave vectors lengths were close to each other and matched with the geometric directions. 

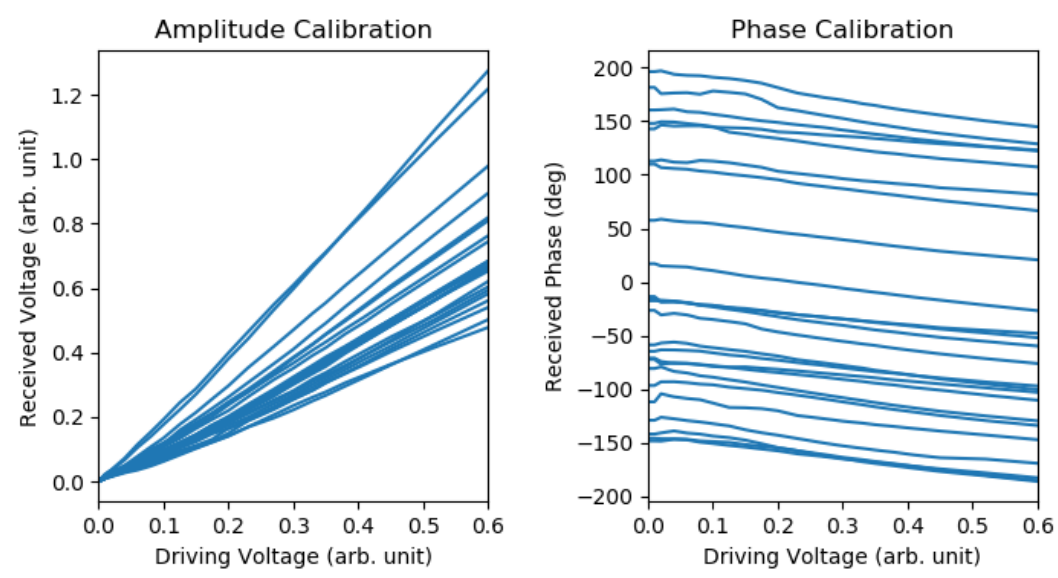

Figure S3: Calibration curves. On left received voltage versus driving amplitude. Larger channels have larger pressure. On right received phase versus driving voltage. The phase shift is inherit transducer property.

\section{Control PCs}

A Raspberry Pi (Model 1B+) was used to drive the SPI-bus and to control the oscilloscope. The Pi was controlled over ssh (secure shell) using a separate laptop where the phase calculation was also done. Both the control and phase calculation software were developed with Python 2.7.

\section{Imaging System}

The samples were imaged during the levitation with a Phantom v611 camera (operated at 100 frames per second (fps) for control samples and 24 fps for the rest, exposure time: $4 \mathrm{~ms}$ ) carrying Navitar long working distance 12x high zoom optics (1-50487AD 12X Body Tube, 1-62922 2x F-mount, 1-50013 0.75x Lens Attachment). The sample was illuminated with two focused infrared LEDs (850 nm, LZ4-40R400, LED Engin) positioned on both sides of the objective.

\section{Samples}

The control samples were elongated polystyrene particles $\left(\rho \approx 20 \mathrm{~kg} / \mathrm{m}^{3}\right)$ with the longest dimension of $5-6 \mathrm{~mm}$. The samples were chosen to have a large aspect ratio between all principal axes. The post mortem fly was $4 \mathrm{~mm}$ long. Its wings were removed prior to the experiment to fit it inside the acoustic trap. The samples were placed in the levitator using tweezers. The sample for time-lapse study was a 5-mm piece of dough (flour, sugar, yeast) initially shaped into an ellipsoid. The sample was slowly $\left(360^{\circ} / 4 \mathrm{~min}\right)$ rotated about the z-axis. No 3D reconstruction was applied to the dough sample as its shape was evolving during the rotation.

\section{Orientation detection}

Detecting three-axis orientation of a complex 3D sample is non-trivial. A computer vision approach based on structure from motion (SfM) was chosen to achieve maximum accuracy and simultaneous quantification for all three angles. The downside was the complexity of the analysis and the amount of tweaking needed. The method requires high resolution pictures of the sample with surface details. It was possible to employ SfM algorithm because the sample orientation was approximately known at all times thanks to the levitation method. This made it possible to supply weak a priori information that helped the reconstruction to converge even with the otherwise challenging image sets.

The SfM algorithm requires a static sample and a moving camera. This limitation was overcome since an orbiting camera produces identical pictures compared to pose obtained from rotating sample with the exception of the static lighting chancing slightly. The trouble with lighting was alleviated by employing small rotation step and by using two light sources. 
Table S1: The reconstruction parameters differing from the OpenSfM defaults.

\begin{tabular}{l|r|r|r|r|r} 
sample & Loss Function & Matching Neighbors & Align Method & Focal Priori & Re-triangulation \\
\hline control 1 & CauchyLoss & 7 & naive & 1.8 & no \\
control 2 & SoftLOneLoss & 5 & naive & 1.8 & no \\
control 3 & SoftLOneLoss & 5 & naive & 1.8 & yes \\
fly & SoftLOneLoss & 9 & naive & 1.8 & no
\end{tabular}

The SfM based orientation detection was implemented with OpenSfM pipeline (v0.1.0) [1]. The pipeline was modified to use the orientation information obtained from the levitation system as a weak prior for the camera location and direction. Each camera location was set on a surface of a sphere (radius $r=50$ ) with OpenSfM degree of precession (dop, the confidence limit for the location prior) set to \pm 6 . As the sample was known to be located at the origin, a weak location a priori condition was also applied to all of feature points found, bounding them to the origin (dop \pm 35 ). The cameras were set to point towards the origin with confidence limits $\pm 10^{\circ}$ also taking into account the tilt.

Initially, the imaging angle was aligned with the samples major axis $\mathbf{b}_{x}$. After applying rotation $R$ on the sample, corresponding movement of the camera was calculated by applying reverse rotation $R^{-1}$ for the initial camera position. Providing the a priori estimates ensured that the reconstruction did not deviate too much from the real geometry. A typical problem without the a priori conditions was that the reconstruction underestimated the curvature and thus the two ends of the circular image sets did not meet. The estimated positions were used by the pipeline to restrict the search for common features to the closest image pairs.

For each control sample reconstruction, a set of 360 images was chosen, 120 around each axis in 3 degree steps. The images were picked from the recorded videos accepting only sharp images (the total optical flow[2] between 10 successive frames was calculated and the one with smallest value was chosen). The reconstruction of the fly was done using a set of 184 sharp images obtained doing rotation about the $z$-axis. Default reconstruction parameters (HAHOG: Hessian Affine feature point detector, Histogram of Oriented Gradients descriptor) were used except for the ones reported in Table S1.

\section{Sample orientation recovery}

The SfM reconstruction minimizes a massively complex object function. In the final solution, the matched features in different images are aligned as well as possible. This minimization determines the local relative position of each image effectively overriding the weak prior information. The global position and orientation of the reconstruction on the other hand is set by the prior conditions which link the detected angles to the intended angles. In an ideal case the orientation detection analysis should be done without the weak a priori conditions to prevent them from affecting the final outcome. This is however not possible because the reconstruction does not converge to the right solution without help.

For the separate three-axis study, the sample orientation angle was calculated from the recovered camera pointing-direction $\mathbf{c}$ (not the camera position) by taking the $\arctan 2\left(\mathbf{c}_{i}, \mathbf{c}_{j}\right)$ of the two vector components perpendicular to the current extrinsic rotation axis. This intentionally limits the study to one angle at a time. The rotation state of the sample has three degrees of freedom and the deviation of the other two angles is hidden here. The off-axis study addresses the full rotation state.

The rest of the analysis (position-wise stability, stability over time, off-axis orientations) was conducted by adding the images to the existing reconstructions one by one while restoring the reconstruction to its original state after each frame. This approach was needed to keep the computation time down and to allow for the the initial reconstruction with a pure set of images without close duplicates. Extending the reconstruction was made possible by further modifying the pipeline with the code in reference [3].

In the position-wise stability study, the stability estimates for Figure 2a (main) were determined. Five additional frames for each rotation were picked and fitted to the initial reconstruction. The angles along the studied axis were calculated and the reported error bar is their standard deviation. The difference between the samples, also illustrated in the same figure, was calculated as the standard deviation of the set of three angles from the three samples. The reported average absolute deviation from desired orientation $\left(3^{\circ} \pm 2^{\circ}\right)$ was averaged over all the samples and all orientations, its limits were calculated as the standard deviation. 
The stability over time was determined for control sample \#2 in a single orientation (yaw $90^{\circ}$, roll $0^{\circ}$, pitch $0^{\circ}$ ). Again the images were fitted to the existing reconstruction and the orientation for each was calculated. Adding an image slightly realigned the model as it settled into a new global minimum. This effect was compensated for to achieve maximum accuracy by comparing the orientation of the newly added image to the nearest existing picture. The orientation was retrieved in $150 \mathrm{~ms}$ steps for the 1-min video and in $10 \mathrm{~ms}$ steps for the 5 -s region of interest (ROI) at $t=35 \mathrm{~s}$. For the ROI dataset, a median filter followed by a third-order Savitzky-Golay filter were applied and the smoothed data was overlaid on top of raw angle data.

The off-axis study, presented in Figure 2c (main), was done for control sample \#3 by running it through a predetermined set of angles stopping for $10 \mathrm{~s}$ in each orientation. The transitions were made in small steps to maintain the orientation. The images obtained from the recorded video were added to the initial model. Some images were discarded as they could not be added since they were taken from an angle where previously found surface features were not recognizable. The three degrees of freedom for the orientation state was visualized in an Aitoff projection plot [5](yaw, pitch) with the roll angle presented as an arrow. The angles were calculated from the local coordinate system of the SfM camera [4], i.e., the orientation of the camera was first calculated by finding the rotation matrix causing the particular camera coordinate base and then calculating the corresponding Tait-Bryan angles [6].

\section{Sample shape recovery}

The point cloud provided by the initial reconstruction was used to derive the 3D surface models. Surface reconstruction and point filtering were done with MeshLab [7]. In case of control samples, the points with dark color (HSV value $<0.3 / 1$ ) were considered as outliers and were removed. Gaussian surface reconstruction was applied to the remaining point cloud. The models provided in Supplementary Data were simplified to reduce their size. The reconstruction of the fly was done in a similar way with the exception that the initial point cloud was manually filtered to remove the outliers, the point-wise normals were recalculated (Filters/Point Set/Compute Normals for Point Sets), and the Gaussian surface reconstruction was applied twice successively to smooth out excessive details of the noisy point cloud.

\section{Simulations}

The Gor'kov potential is only an approximate method for studying the forces acting on a levitated sample. To calculate the torque, a more sophisticated analysis is required. This can be done by solving the scattering problem numerically and by evaluating the momentum flow carried out by the acoustic waves. To achieve this, the experiment was recreated with a finite element method (FEM) modeling software COMSOL Multiphysics ${ }^{\circledR}$ version 5.2. The torque was calculated from first order harmonic acoustic perturbation which was solved with the Pressure Acoustics Module in the frequency domain.

The simulation geometry for a levitated control sample was obtained with the SfM. The 3D surface model was imported into the simulating software and was scaled to match its real-world dimensions. Due to the complex 3D shape, the simulation had to be done in 3D and no symmetries could be exploited. The sample was surrounded by a spherical $(r=1.6 \lambda)$ air (built-in material, $T=20^{\circ} \mathrm{C}$ ) domain. The boundary between the sample and air domains was set as sound hard boundary due to the large difference in the acoustic impedance. The air domain was truncated using a perfectly matched layer (PML, thickness $0.5 \lambda)$ that absorbs the scattered waves. The incident wave was created using a background pressure field as the two perpendicular standing waves introduced in Eq. S3 $\left(\alpha=0.4, p_{0}=2 \mathrm{kPa}\right)$. The geometry was meshed with free tetrahedral elements (with the exception of the PML that was swept radially) with maximum element size $\lambda / 8$ (quadratic Lagrangian elements).[8]

The simulation was repeated for sample orientations along the main rotation axes to obtain the torque as a function of orientation. To avoid re-meshing, the direction of the incident waves was changed instead of rotating the sample domain. The sample was initially set to the estimated equilibrium position and orientation.

The torque acting on the sample was evaluated in each orientation by calculating the acoustic radiation force on the sample surface $S[9]$ :

$$
\langle\mathrm{d} \mathbf{F}\rangle=\left(\frac{\rho}{2}\left\langle V^{2}\right\rangle \mathbf{n}-\frac{1}{2 \rho c^{2}}\left\langle P^{2}\right\rangle \mathbf{n}-\rho\langle(\mathbf{n} \cdot \mathbf{V}) \mathbf{V}\rangle\right) \mathrm{d} S .
$$


Due to the conservation of angular momentum, it is enough to study the integral on any enclosing surface [10], where the integral is numerically stable. The time-averaged torque $\langle\boldsymbol{\tau}\rangle$ can be written as:

$$
\langle\boldsymbol{\tau}\rangle=\oint_{S_{e}} \mathbf{r} \times\left(\frac{\rho}{2}\left\langle V^{2}\right\rangle \mathbf{n}-\frac{1}{2 \rho c^{2}}\left\langle P^{2}\right\rangle \mathbf{n}-\rho\langle(\mathbf{n} \cdot \mathbf{V}) \mathbf{V}\rangle\right) \mathrm{d} S,
$$

where $\mathbf{V}=\operatorname{Re}[\mathbf{v}]$ is the particle velocity and $P$ the acoustic pressure. The integral was evaluated in the simulation software on a spherical enclosing surface $(r=0.8 \lambda)$. Due to the choice of the spherical surface $S_{e}$, the two first terms can be omitted $\left(\mathbf{r}_{S_{e}} \times \mathbf{n}_{S_{e}}=0\right)$. The remaining term must be written using the complex presentation rather than the time average $(\langle(\mathbf{n} \cdot \mathbf{V}) \mathbf{V}\rangle)$ to be compatible with the FEM frequency domain solution for velocity field $\mathbf{v}$.

$$
\langle\boldsymbol{\tau}\rangle=-\rho \oint_{S_{e}} \mathbf{r} \times \frac{1}{2} \operatorname{Re}\left[\left(\mathbf{n} \cdot \mathbf{v}^{*}\right) \mathbf{v}\right] \mathrm{d} S
$$

The torque curves exhibited the expected sinusoidal shape and estimated a realistic magnitude of $10 \mathrm{nNm}$. The curves for pitch and yaw were slightly distorted which is explained by the finite length of the particle in the $\mathbf{b}_{x}$ direction $(>0.5 \lambda)$.

\section{References}

[1] Open source Structure from Motion pipeline https://github.com/mapillary/OpenSfM, accessed 11/2018

[2] Optical Flow, Open Source Computer Vision https://docs.opencv.org/3.4/d7/d8b/tutorial_py_lucas_kanade.html, accessed 11/2018

[3] OpenSfM reposity pull request "Allow reconstructions to be extended" https://github.com/mapillary/OpenSfM/pull/178, accessed 11/2018

[4] OpenSfM Documentation, Release 0.1.0 https://opensfm.readthedocs.io/en/latest/cam_coord_system.html, accessed 11/2018

[5] Geographic Projections, Matplotlib Documentation, accessed 11/2018 https://matplotlib.org/gallery/subplots_axes_and_figures/geo_demo.html

[6] Diebel, James. "Representing attitude: Euler angles, unit quaternions, and rotation vectors." Matrix 58.15-16 (2006): 1-35.

[7] Cignoni, Paolo, et al. "Meshlab: an open-source mesh processing tool." Eurographics Italian chapter conference. Vol. 2008. 2008.

[8] Comsol Multiphysics ${ }^{\circledR}$ Acoustic Module User Guide

[9] Bruus, H. Theoretical microfluidics. Vol. 18. Oxford: Oxford university press, 2008.

[10] Wijaya, Felix Bob, and Kian-Meng Lim. "Numerical calculation of acoustic radiation force and torque acting on rigid non-spherical particles." Acta Acustica united with Acustica 101.3 (2015): 531-542. 